\title{
Image Set Creation using different Image Enhancement Technique for Underwater Image Segmentation
}

\author{
Vikram Dwivedi \\ Department of Electronics \& \\ Communication \\ TCST Bhopal India
}

\author{
Paresh Rawat, PhD \\ Department of Electronics \& \\ Communication \\ TCST Bhopal India
}

\author{
Nashrah Fatima \\ Department of Electronics \& \\ Communication \\ TCST Bhopal India
}

\begin{abstract}
Underwater images suffer from less visibility problem due to principal of light refraction under water environment. Therefore, this paper address the problem of enhancing the contrast of the underwater images captured under different lightning conditions and depths. This paper presenting the comparison of the two distinct popular enhancement methods and it concluded that this different method generates two different image sets from original low contrast image. These sets can be further utilized for object segmentation using fuzzy based clustering methods efficiently. Paper presents enhancement using contrast limit adaptive histogram equalization (CLAHE) and, global contrast adjustment method.. The CLAHE method is used to the images so that contrast of output images is improved also gives better information. It is concluded that using the different contrast enhancement method different image data set can be created for same input image which can be utilized for higher level processing task and features extractions.
\end{abstract}

\section{General Terms}

Image processing, Underwater Images,

\section{Keywords}

Contrast enhancement, Contrast adjustment, Contrast limited Adaptive Histogram Equalization, Dual enhancement, Entropy

\section{INTRODUCTION}

In the past years uses of underwater objects have increased significantly. A lot of work has done in the area of image enhancement but in case of underwater images it is more applicable due to there is a problem of accurateness of underwater images. The quality of underwater image is degraded due to these effects of scattering of light, absorption, reflection and banding losses etc. The various reasons of the low contrast in under water images are shown in Figure 1 Incident, reflected and refracted light rays are shown by different colors in Figure 1.

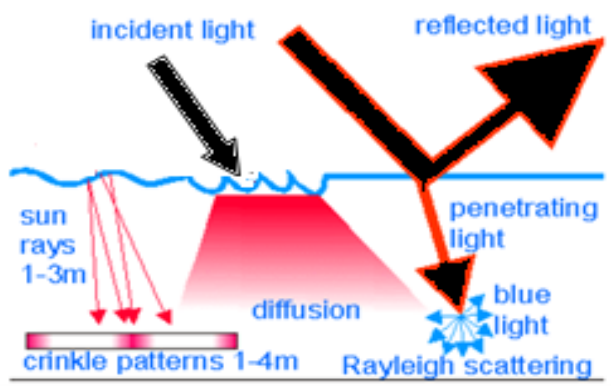

Figure 1 Reasons of low contrast in underwater environment
Enhancing the contrast is the most common method of enhancing the images [8].The image contrast is the difference in the color levels[11].The researchers have invented many image enhancement techniques Viz. Contrast adjustment, contrast limit adaptive histogram equalization(CLAHE) contrast stretching etc..We are using contrast limit adaptive histogram equalization and contrast adjustment methods. This paper further describes the various authors who gave their views in image enhancement. Section 3 describes the contrast adjustment [7] method. The section 4 describes the contrast limit adaptive histogram equalization method. Section 5 describes the process dual enhancement technique [12].In section 6 the result are presented which are expected outcome of the experiment. Section 7 describes the conclusion and future work.

\section{LITERATURE REVIEW}

There are many enhancement techniques in which intensity enhancement is important method. In which CLAHE [14] and histogram equalization [15] methods are popular ones..Both of these methods are intensity based. Intensity based methods are spatial domain methods. There are two types of enhancement methods spatial domain methods and transform domain methods. K.K. Sharma et al. [8] have presented color image enhancement in transform domain using non linear mapping. Harish et al. [5] have used discrete wavelet transform based methods for color enhancement. But under the underwater environment due to non uniform lightning transform based color enhancement are having probabilistic performance. Thus this paper focuses on spatial domain enhancement methods which are less complex too.

Constantim Vertanhave [9] proposed to use Histogram equalization of colour images using the adaptive neighbourhood. But method was color sensitive. Paresh Rawat ,Jyoti Singhai [15] in their previous work have presented various histogram based contrast enhancement methods.

Etaa D. Pisano et al [7] have suggested using contrast limited adaptive histogram equalization (CLAHE) method for improving the image quality. Various researchers used CLAHE as tool for pre processing task as [7, 11 and 14]. Rafael Garcia et.al, studied different local histogram equalization methods and its variations. They have also discussed CLAHE enhancement method in their research in order to solve lighting problems in underwater imaging. Antonis Daskalakis [11] et.al, have proposed an efficient spotadaptive CLAHE based image segmentation technique to improuve microarray gènes quantification. They found that this technique improved the display of spots and emphasized on the depiction of spots. Hitam et al. [9] have presented a combination of the CHAHE in RGB and HSV colour spaces for underwater images. 
Among all spatial domain methods CHAHE is less affected by colour and brightness shifting problem thus widely used.

\section{CONTRAST ADJUSTMENT}

Contrast adjustment means the adjustment of contrast in the images. The peak of the intensity levels is increased or adjusted in this contrast adjustment. Contrast adjustment is done to improve the image in terms of brightness and better visibility. In this paper this is applied in underwater images [15]. Contrast adjustment method maps the image intensity values in gray image I to modified image values $\mathbf{J}$ so that $1 \%$ of data is saturated at low and high intensities of then image I. This in turn increases the contrast of the output image J. this method is similar to the contrast stretching within the limit. For the color images the contrast is adjusted separately in RGB color space. Contrast adjustment method is implemented in two steps;

1. Observe histogram of the image and determine the limits of intensity value.

2. Convert these limits as a fraction within 0.0 and 1.0 and pass them to imadjust function in the [low_in high_in] vector.

\section{CONTRAST LIMITED ADAPTIVE HISTOGRAM EQUALIZATION}

Method is also known as CLAHE is a enhancement method is which gives the optimal equalization and also limit the image contrast. This method is very popular for enhancing the images in the underwater environment. Contrast Limited Adaptive Histogram Equalization (CLAHE) method is basicale an improved formé of Adaptive Histogram Equalization (AHE). Method overcomes the limitations of standard histogram equalization. Noise can be reduced while maintaining the high spatial frequency content of the image by applying a combination of CLAHE, median filtration and edge sharpening.

CLAHE method sub divides an image in to small regions called tiles, instead of entire image. Each tile's contrast is enhanced, so that the histogram of the output region approximately matches the histogram specified by the distribution types. The step wise algorithm is explained as follows;

1. Read the underwater color image.

2. Now after this convert the image into RGB color space.

3. Image size is $240 * 320$.

4. Divide the image into different color spaces Red, green and blue colors.

5. Now after dividing them save these color spaces into different variables.

6. Now divide the image into small partitions.

7. These small partitions are called tiles.

8. Now after partioning calculate the probability distribution of the gray levels.

9. Now calculate the modified gray levels which are modified.

10. The gray level is calculated in each tiles, separately.

11. For standard CLAHE method with uniform distribution can be given as

$$
g=[g \max -g \min ] * p(f)+g \min
$$

where gmax $=$ maximum gray level value

gmin=Minimum gray value

$\mathrm{g}$ is the computed pixel vaue

$\mathrm{p}(\mathrm{f})=\mathrm{CPD}($ Comulative probability distribution $)$ for exponential distribution gray level can be adapted as

$g=\operatorname{gmin}-(1 / a)$ alpha $* \operatorname{in}[1-p(f)]$

Where alpha is clipping parameter.

12. In this paper the uniform distribution is used. Since it is needed for applications like segmentation.

13. Using the difference gmax-gmin improves the image conytrast

14. Then gmin is adaptively to cumulative distribution.

15. Plot the image which is enhanced and draw the histogram.

16. End of the algorithm. Here algorithm is finished..

17.The CLAHE method only stretches the contrast range ,it also optimizes the entropy of the image, Thus it is widely used for the high level applications such as segmentation and object detection.

Thus the CLAHE method not only stretches the contrast range but also optimizes the entropy of the image. Thus is widely used for the high level applications such as segmentation and object detection.

\section{DUAL ENHANCEMENT METHOD}

In this paper it is proposed to enhance the images using combination of CLAHE method and contrast adjustment method. Therefore method is named as dual enhancement technique. It is observed that adjusting the image contrast after enhancing the image using CLAHE method may improve the efficiency of the higher level processing methods.

\section{EXPERIMENTAL RESULTS}

In this section, some experimental results of performance comparison for various contrast based enhancement method are presented.

\subsection{Input Images Used}

The input underwater images in Figure 2, are taken from different under water environments. All images suffer from the low and poor contrast due to underwater environment and containing the different kind of objects. For analyzing all the images are resized to $240 * 320$. The histogram of the images is given below in Figure 3. It can be observed from Figure that each image contains different objects and information.
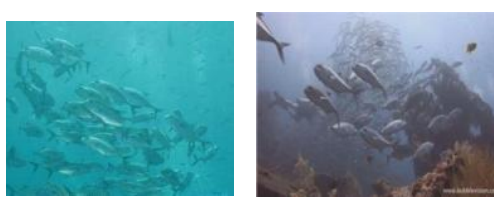

a) See image b) Bubble_vision image

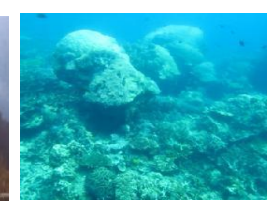

c) Redang_Island_2
Figure.2 Original input images used in the paper 


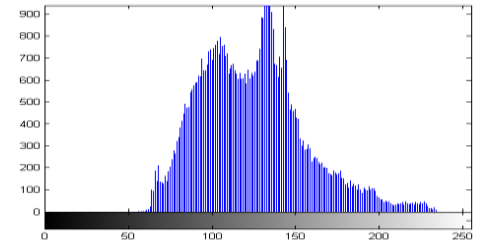

a) Bedang_island_2

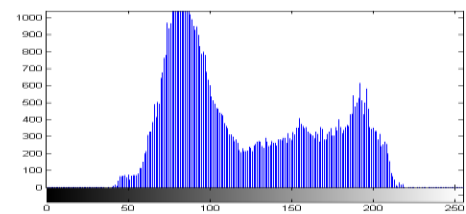

b) Bubble_vision_image

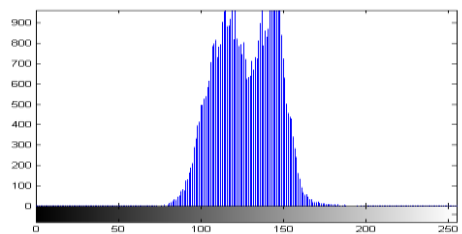

c) See.image

Figure 3 Histogram of input images
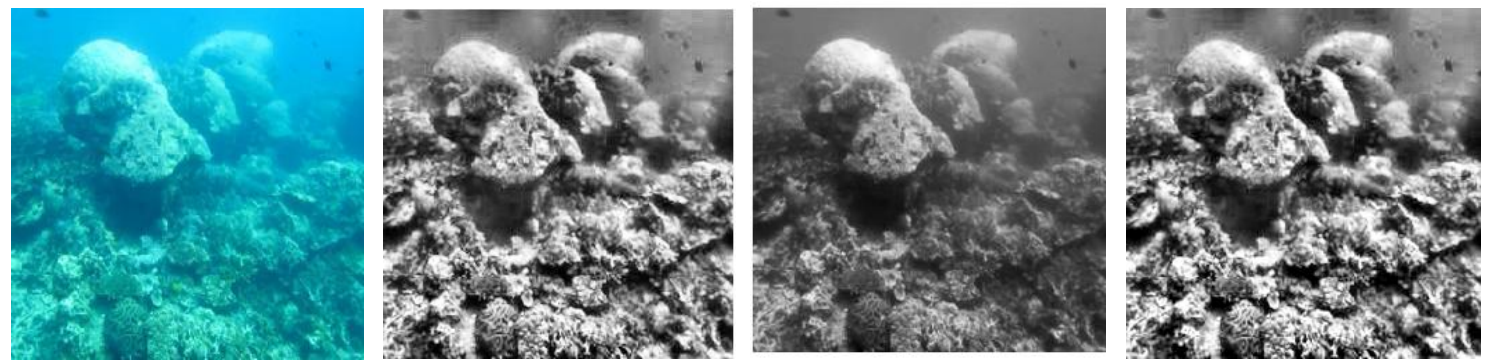

a) Original image of the Redang_island b) clahe enhanced image c) Contrast adjustment d) Dual Enhancement
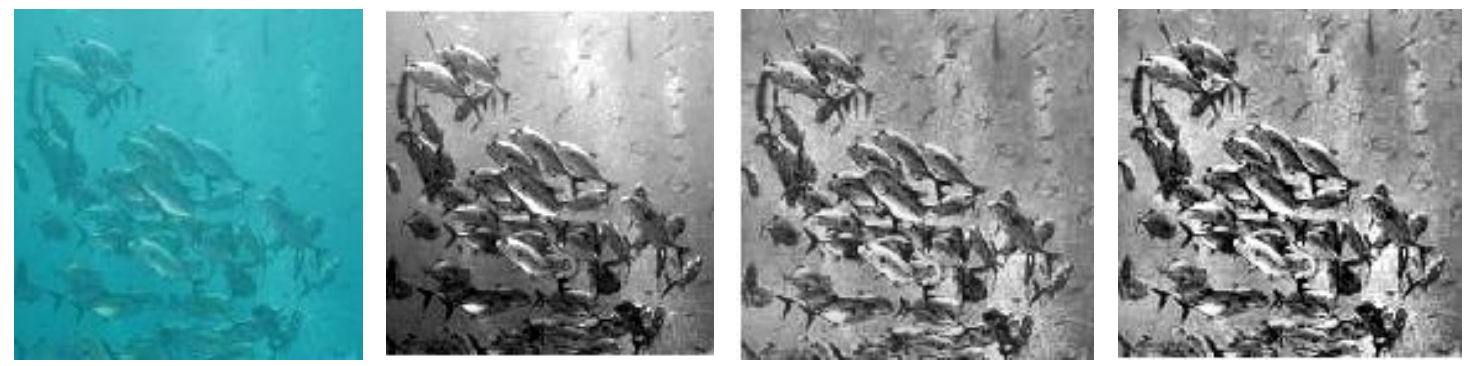

d)

Original See image

e) CLAHE enhanced image f) contrast adjustment g) Dual Enhancement
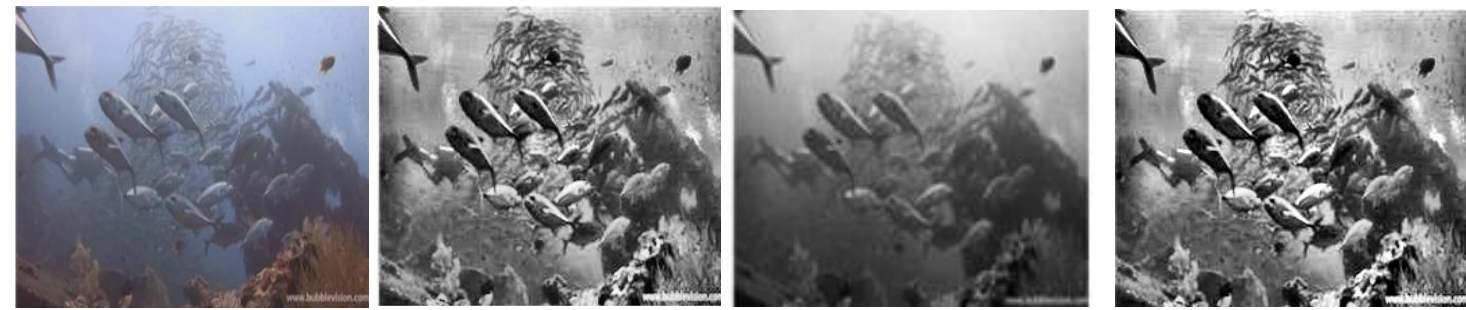

h) Original Bubble_vision image

i) CLAHE enhanced image j) contrast adjustment $\mathrm{k}$ )

Figure 4 Result of the image set generation using various enhancement methods a) Column one is original images b) Column two shows enhanced CLAHE images and c) column three is Contrast adjust image d) Column four is Dual enhanced image
6.2.1. Discrete Entropy)

The entropy is defined as the measure of the information in the image. More the entropy more is the information. In the discrete form entropy may be defined as; 


$$
h(x)=\sum_{i=1}^{m x} f(x) \log (f(x)) d x
$$

Table 1 Comparison of Entropy

\begin{tabular}{|c|c|c|c|c|c|}
\hline $\begin{array}{r}\text { S. } \\
\text { No }\end{array}$ & Images & Original & $\begin{array}{c}\text { With Contrast } \\
\text { adjusted }\end{array}$ & CLAHE & $\begin{array}{c}\text { With } \\
\text { Dual } \\
\text { method }\end{array}$ \\
\hline 1 & $\begin{array}{c}\text { See } \\
\text { image }\end{array}$ & 6.118 & 7.830 & 7.403 & 7.679 \\
\hline 2 & $\begin{array}{c}\text { Bobble } \\
\text { vision } \\
\text { image }\end{array}$ & 7.117 & 7.757 & 7.688 & 7.8548 \\
\hline 3 & $\begin{array}{c}\text { Redang } \\
\text { Island }\end{array}$ & 6.916 & 7.6045 & 7.8689 & 7.9147 \\
\hline
\end{tabular}

The parametric comparison of the entropy for underwater image enhancement methods is presented in the Table 1. It can be observed that performance of the contrast adjust method degrades in terms of entropy for Bubble_vision image while the performance of the CLAHE enhancement degraded

for See image. The performance of the Dual enhancement method with the sequential combination of CLAHE and contrast adjustment method improves the entropy and thus contains more information.

\section{CONCLUSION}

CLAHE gives better results than any other methods in field of contrast enhancement. CLAHE gives higher Entropy. Presence of noise is less in such enhanced images. CLAHE method is used in underwater image because it gives better visibility for underwater images. But due to its color sensitivity CLAHE methods performance in terms of entropy degrade for few images and it may cause information loss. In this paper it is proposed to use the dual method of adjusting the contrast after CLAHE enhancement. This method improves contrast and also improves the entropy of the image significantly. It is found that each enhancement method gives the different image for the same input image thus multifocused data set can be generated.

\section{ACKNOWLEDGMENTS}

Author wishes to acknowledge each and every individual who have supported for the current work directly or indirectly.

\section{REFERENCES}

[1] G. Nikifordis, I. Kalatzis , D. Cavouras, P. Bougioukos, S. Kostopoulos, A. Daskalakis,."An efficient Clahebased, spot adaptive image segmentation technique for improving microarray genes' quantification", Research gate 2012

[2] Ravichandran, C.G. , V. Magudeeswaran, "An Efficient Method for Contrast Enhancement in Still Images using Histogram Modification Framework", Journal of Computer Science Vol. 8 No. 5: PP. 775-779, 2012.

[3] Y.-T. Kim Contrast Enhancement Using Brightness Preserving Bi-Histogram Equalization , IEEE Transactions on Consumer Electronics, Vol. 43, No. 1, FEBRUARY 1997

[4] Y.Wang, Q. chen, B. zhang, "Image enhancement based on equal area dualistic sub-image histogram equalization method", IEEE Transaction of Consumer Electronics Vol. 45, Issue 1, PP 68-75 Feb 1999.
[5] Harish parthsarthy,Tarun K. Rawat ,Prateek S. Sengar ,"Color image enhancement by scaling the discrete wavelet transform coefficients."International Conference on Microelectronics, Communications and Renewable Energy (AICERA/ICMiCR), 2013

[6] Etta D, Pisano, S. Zong, R. E Jhonston "Contrast limited adaptive histogram equalization image processing to improve the detection of simulated speculation in Dense Monograms", Journal of Digital Imaging, vol. 11, No. 4, pp 193-200, 1998

[7] Constantim Vertan ,"Histogram equalization of colour images using the adaptive neighbourhood approach",

[8] Muhammad Suzuri Hitam,. Wan Nural Jawahir Hj Wan Yussof, Ezmahamrul Afreen Awalludin,, Zainuddin Bachok, "Mixture Contrast Limited Adaptive Histogram Equalization for Underwater Image Enhancement",

[9] M. Abdullah-Al-Wadud, M. H. Kabir, M. A. A. Dewan, and Oksam Chae, "A dynamic histogram equalization for image contrast enhancement", IEEE Trans. Consumer Electronics, vol. 53, no. 2, pp. 593 - 600, May 2007.

[10] Manpreet Kaur, Jasdeep Kaur, Jappreet Kaur, “Survey of Contrast Enhancement Techniques based on Histogram Equalization", (IJACSA) International Journal of Advanced Computer Science and Applications, Vol. 2, No. 7, 2011

[11] S. A. Hariprasad, Arunlal. K. S, Vikhar Ahmed, "Novel Weighted Mean Separated Histogram Equalization for Contrast Enhancement of Underwater Images", International Journal of Scientific \& Engineering Research, Volume 4, Issue 12, December-2013.

[12] K. K. Sharma, Supriya M.," Color Image Enhancement using Nonlinear Mapping in Color and Transform Domains", International Journal of Advanced Electronics \& Communication Systems, Issue 2 Vol. 1 2012.

[13] Muhammad Suzuri Hitam,Wan Nural Jawahire Huj Wan Yussof,Ezmahamrul Afreen Awalludin, Zainuddin Bachok,"Mixture Contrast Limited Adaptive Histogram Equalization For Underwater Image Enhancement .

[14] Jyoti Singhai, Paresh Rawat, "Image Enhancement Method for Underwater, Ground and Satellite Images Using Brightness $\hat{A}$ Preserving Histogram Equalization with Maximum Entropy," iccima, vol. 3, pp.507-512, 2007 International Conference on Computational Intelligence and Multimedia Applications, 2007

\section{AUTHOR PROFILE}

1. Vikram Dwivedi: have completed the BE in Electronics and Communication engineering and is currently pursuing $\mathrm{M}$. Tech degree from Truba college Bhopal India.

2. Nashrah Fatima: Have received M. Tech degree and is currently working as Astt. Prof at Truba College Bhopal in Electronics and communication department

3. Dr. Paresh rawat Have received $\mathrm{PhD}$ degree in the Electronics and communication (Video processing) field from the MANIT Bhopal, and is currently working as Head of the department ECE Truba College Bhopal India 